\title{
A collaborative effort to build an epilepsy program in central Vietnam
}

The Socialist Republic of Vietnam is a developing country with an estimated population of 96.2 million as of 2019. The prevalence rate of epilepsy is between 4.4 and 14 per 1000 population. ${ }^{1,2}$ The care of epileptic patients in Vietnam is suboptimal for many reasons: insufficient resources, poor infrastructure, lack of education (to both patients and physicians), and inadequate offer of medical and surgical treatment options for epilepsy. ${ }^{3}$

Before 2012, there was no comprehensive epilepsy center in Vietnam. ${ }^{4}$ Here, we described our experience in improving epilepsy care in a central province of Vietnam with minimal resources through a collaboration which was established between Hue University of Medicine and Pharmacy (HUMP) and Centre Hospitalier de l'Université de Montréal (CHUM).

The HUMP located in Thua Thien Hue Province has the role of training medical doctors for central provinces and Tay Nguyen highlands of Vietnam, but it has minimal resources for epilepsy. Before 2012, there was no epileptologist, epilepsy neurosurgeon, video-EEG machine, nuclear medicine neuroimaging, and only a 0.23 Tesla MRI machine. Hence, we started to collaborate with CHUM to improve the medical and surgical care of patients with epilepsy in the central provinces of Vietnam.

\section{CHUM-HUMP collaborative work}

Several measures have been taken since 2012. A neurologist and a neurosurgeon from HUMP began a two-year fellowship in epilepsy at CHUM. Organized jointly by the CHUM and HUMP, epilepsy symposia were held to improve the knowledge and practices of health professionals caring for people with epilepsy in Central Vietnam. The first symposium was held in March 2013, which attracted 150 participants who benefited from an update on the epilepsy diagnosis and management (Figure 1). The second symposium focused on clinical practice and hands-on training workshops on electroencephalography. The third symposium was organized combining lectures and practical bedside teaching of physicians by seeing local epileptic patients with them in 2015 (Figure 2). During this symposium, the CHUM epilepsy group proceeded with the donation of a new Nihon Kohden videoEEG machine and the inauguration of the HUMP epilepsy clinic. Continued support is ongoing via telehealth, a series of 14 recorded educational webinars based on the ILAE curriculum, and in-person symposia has been held every two years.

\section{The challenges}

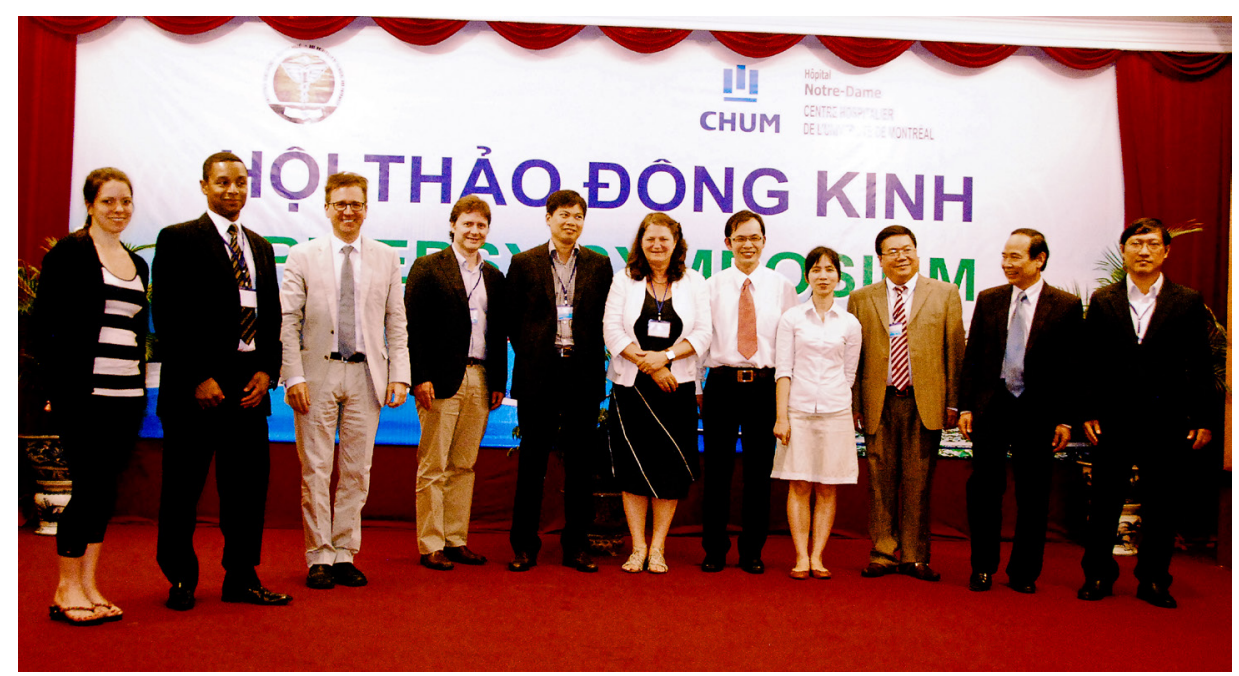

Figure 1. Epilepsy symposium organized jointly by the CHUM and HUMP. 


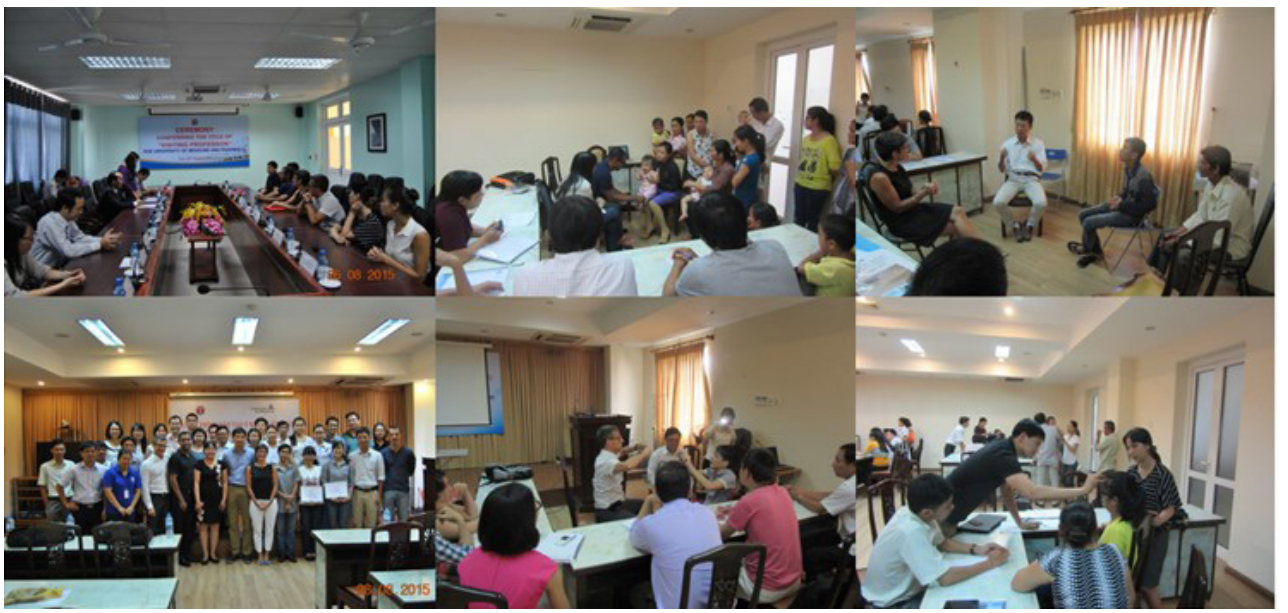

Figure 2. Epilepsy symposium combining lectures and practical bedside teaching

The most significant challenge our group encountered throughout this collaborative endeavor has been Vietnamese physicians' skepticism towards epilepsy surgery; explaining the low-referral rate at the HUMP epilepsy clinic for surgical treatment. Although the role of surgery in intractable epilepsy patients was discussed with the participants through the joint symposia and consultation, it is likely that a significant change in practice will only come with local demonstrations of epilepsy surgery's efficacy by the HUMP epilepsy team with actual cases.

Another major challenge for the HUMP epilepsy team was the lack of long-term EEG recording. Because of the limitation in personnel and lack of a bed on the ward, overnight EEG recordings were impossible, and the team had to resort to 10-hour-long video-EEG recordings during the day. This made it more difficult to record the epileptic seizures, ictal discharges and localize the seizure focus. Finally, lack of surgical equipment was (and still is) a challenge for the HUMP team. For example, the neurosurgeon has to operate with an old Leica surgical microscope, which has only one binocular, but no cable connected to the video screen (Figure 3). As a result, the assistant and scrubbing nurse have difficulty assisting him, not being able to see what he is doing.

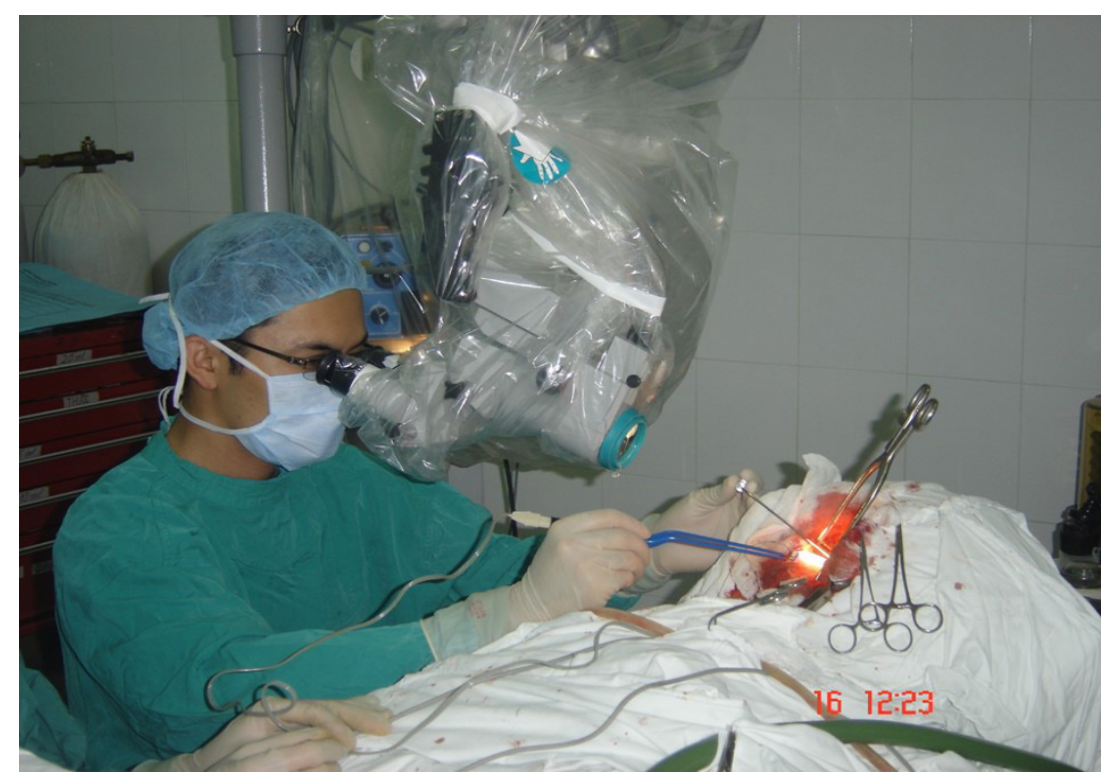

Figure 3. Epilepsy surgery was performed with an old microscope 


\section{Outcomes}

Despite these challenges, this collaborative work between the CHUM and the HUMP has led to some concrete achievements.

The first epilepsy surgery was performed in December 2016. A patient with drug-resistant epilepsy and right mesial temporal sclerosis benefited from an anterior temporal lobectomy which provided seizure freedom (follow up of 4 years). Subsequently, two corpus callosotomies was performed for 2 patients with drop attacks associated with Lennox-Gastaut syndrome. The two patients are Engel 1a for atonic seizures postoperatively (follow up of 3-4 years). In 2018, 4 anterior temporal lobectomies and 1 frontal lobectomy for 5 patients with lesional epilepsy were performed. All of them were Engel 1 post-op (follow up of 3 years).

Knowledge transfer by the CHUM epilepsy team to participants at symposia mentioned above and the success of epilepsy surgery have led to an increase in referrals to the HUMP epilepsy clinic since 2015.

The HUMP epilepsy team, in their turn, with the help of CHUM team, will train the Vietnamese physicians to build epilepsy programs in other hospitals in Vietnam.

In conclusion, the successful collaboration between CHUM and HUMP is a model that can be replicated to develop epilepsy care in developing countries with minimal resources.

${ }^{1}$ Thi Phuoc Yen Tran, ${ }^{2}$ Van Tri Truong, ${ }^{1}$ Dinh Toan Nguyen, ${ }^{1}$ Duy Duan Nguyen, ${ }^{3}$ Alain Bouthillier, ${ }^{4}$ Dang Khoa Nguyen

${ }^{1}$ Department of Internal Medicine, ${ }^{2}$ Department of Neurosurgery, Hue University hospital, Hue University of Medicine and Pharmacy, Hue University, Vietnam; ${ }^{3}$ Division of Neurosurgery, ${ }^{4}$ Division of Neurology, Centre Hospitalier de l'Université de Montréal, University of Montreal, Montreal, Canada

Address corresponding to: Van Tri TRUONG MD, MSc, Department of Neurosurgery, Hue University hospital, Hue University of Medicine and Pharmacy Hue University, 41 Nguyen Hue Street, Hue City, Vietnam. Email: drtruongtri@ gmail.com

https://doi.org/10.54029/2021zsu

\section{DISCLOSURE}

Conflicts of interest: None

\section{REFERENCES}

1. Tuan NA, Cuong LQ, Allebeck P, Chuc NTK, Persson HE, Tomson, T. The prevalence of epilepsy in a rural district of Vietnam: a population-based study from the EPIBAVI project. Epilepsia 2008; 49(9): 1634-7.

2. Mac TL, Tram DS, Quet F, Odermatt P, Preux PM, Tan CT. Epidemiology, aetiology, and clinical management of epilepsy in Asia: a systematic review. Lancet Neurol 2007; 6(6):533-43.

3. Aydemir N, Trung DV, Snape D, Baker GA, Jacoby A, CREST Study Team Multiple impacts of epilepsy and contributing factors: findings from an ethnographic study in Vietnam. Epilepsy Behav 2009; 16(3): 512-20.

4. Lim KS, Chia ZZ, Myint MZ, et al. Epilepsy in Southeast Asia, how much have we closed the management gap in past two decades? Neurol Asia 2020; 25(4): 425-38. 